\title{
Supercurrent and noise in point contact between two different superconductors
}

\author{
S. K. Yip \\ Institute of Physics, Academia Sinica, Nankang, Taipei 11529, Taiwan
}

\begin{abstract}
We show that, for a quantum point contact between two superconductors of different gap magnitudes, Andreev bound states do not exist for certain phase differences and gap ratios. Continuum states may dominate the transport, and the supercurrent noise is qualitatively different from the case of equal gaps.
\end{abstract}

PACS numbers: 74.80.Fp, $74.50+\mathrm{r}$

With nanotechnology an emerging subject, there has been much effort in understanding electrical transport in quantum point contacts [1]. A particular interesting sub-field is the study of contacts between two superconductors. Their transport properties such as currentphase $(I-\phi)$ relationships and current-voltage dependences have been studied theoretically $[2-4]$ as well as measured experimentally $[5,6]$. There are further theoretical works on the important issue of current fluctuations (noise). [4,7-9] All these theoretical papers are for the case where the two superconducting gaps $|\Delta|$ are equal. In the physical pictures developed in these papers, a particularly important role is played by the Andreev bound states that form between the two superconductors. For a short junction with a single conduction channel of transmission probability $D$, there is a pair of bound states at $\pm \epsilon_{b}$ where $\epsilon_{b}=|\Delta|\left[1-D \sin ^{2}(\phi / 2)\right]^{1 / 2}$. [10,11] Here $\phi$ is the phase difference between the two superconductors. In equilibrium, the net supercurrent $I$ is carried entirely by this bound state, and at zero temperature, $I$ is given simply by $I=-\frac{2 e}{\hbar} \frac{\partial \epsilon_{b}}{\partial \phi}$, while at a finite temperature $T$, 
$I$ is reduced by a factor tanh $\left(\frac{\epsilon_{b}}{2 T}\right)$ arising from the occupation numbers at $\pm \epsilon_{b}$. The response of these bound states also play an important role in determining the non-equilibrium electron transport such as $I V$ characteristics and the junction behavior under microwave irradiation. [2-4]

These Andreev bound states also play a major role in determining the supercurrent noise of the point contact. [4,7-9] An important source of the equilibrium supercurrent noise arises from the fact that these bound states can either be occupied or empty due to thermal fluctuations. $[7,8]$ As a result, there are two strong peaks of the current noise at zero frequency and $\omega=2 \epsilon_{b}$.

In this paper we study the bound state, current-phase relationship, as well as noise of a quantum contact between two superconductors with unequal gaps. We shall show that, in some regions of parameter space, the situation is dramatically different from the case where the gaps are of equal magnitude. For certain gap ratios and phase difference, the Andreev bound states do not exist at all. Instead of having bound state carrying all the current, the continuum states with energy between the two gaps can carry a substantial, sometimes even the dominating, part of the supercurrent. Interestingly the total supercurrent, and hence the current-phase relationship, is not significantly changed (except the overall magnitude) even in the case of unequal gaps. However, when the bound states do not exist, the supercurrent noise is qualitatively different. In particular, there is a substantial reduction in noise level for all frequencies below twice the smaller gap. The zero frequency and $2 \epsilon_{b}$ peaks are entirely absent. It should be possible to perform measurements such as STM with a Pb tip on Al to verify the predictions in the present work.

We shall then consider two s-wave superconductors in contact through a barrier with transmission coefficent $D$. Without loss in generality we shall take the left (right) superconductor with smaller (larger) gap magnitude $\left|\Delta_{l}\right|\left(\left|\Delta_{r}\right|\right)$, and take the phase difference $\phi$ between 0 and $\pi$. One can easily adapt the quasiclassical formalism [12] to the present (one conduction channel) case, and obtain the Green's functions $\hat{g}_{l, r}(p, \epsilon)$ and $\hat{g}_{l, r}(\underline{p}, \epsilon)$ for 
positive $(p)$ and negative $(\underline{p}=-p)$ momenta and at a point near and to the left and right (subscript $l$ and $r$ ) of the barrier. It is convenient to express the answers [12,13] in terms of $\hat{s}_{l, r} \equiv \hat{g}_{l, r}(p)+\hat{g}_{l, r}(\underline{p})$ and $\hat{d}_{l, r} \equiv \hat{g}_{l, r}(p)-\hat{g}_{l, r}(\underline{p})$. One obtains $\hat{d}_{l}=\hat{d}_{r}=\hat{d}$ with

$$
\hat{d}=\frac{i D}{2 \pi}\left[\hat{g}_{r, \infty}, \hat{g}_{l, \infty}\right] / \mathcal{D}
$$

and

$$
\hat{s}_{l}=\left((2-D) \hat{g}_{l, \infty}+D \hat{g}_{r, \infty}\right) / \mathcal{D}
$$

where $\mathcal{D}(\epsilon) \equiv 1+\frac{D}{4 \pi^{2}}\left(\hat{g}_{l, \infty}-\hat{g}_{r, \infty}\right)^{2}$. Here $\hat{g}_{l, r, \infty}=-\pi \frac{\epsilon \hat{\tau}_{3}-\hat{\Delta}_{l, r}}{\sqrt{\left|\Delta_{l, r}\right|^{2}-\epsilon^{2}}}$ are the quasiclassical Green's function at positions far away from the interface into the left (right) superconductor. The above equations hold for both the retarded $(R)$ and advanced $(A)$ Green's functions with $\epsilon \rightarrow \epsilon \pm i 0_{+}$and we have left out their superscripts temporarily for simplicity. Here $0_{+}$is an infinitesimal positive number. Explicitly, we have

$$
\hat{d}=i \pi D \frac{\epsilon \hat{\tau}_{3}\left(\hat{\Delta}_{r}-\hat{\Delta}_{l}\right)-i\left|\Delta_{r} \| \Delta_{l}\right| \sin (\phi) \hat{\tau}_{3}}{Q(\epsilon)}
$$

where $Q(\epsilon) \equiv \sqrt{\left|\Delta_{l}\right|^{2}-\epsilon^{2}} \sqrt{\left|\Delta_{r}\right|^{2}-\epsilon^{2}} \mathcal{D}(\epsilon)$, i.e.

$$
Q(\epsilon)=\left(1-\frac{D}{2}\right) \sqrt{\left|\Delta_{l}\right|^{2}-\epsilon^{2}} \sqrt{\left|\Delta_{r}\right|^{2}-\epsilon^{2}}-\frac{D}{2}\left(\epsilon^{2}-\left|\Delta_{r}\right|\left|\Delta_{l}\right| \cos (\phi)\right)
$$

Andreev bound state exists when

$$
Q(\epsilon)=0
$$

for some $|\epsilon|<\left|\Delta_{l}\right| \leq\left|\Delta_{r}\right|$. The solutions to eq (5) always come in $\pm \epsilon_{b}$ pairs and we shall focus on $\epsilon_{b}>0$. Eq (5) can be rewritten as a quadratic equation in $\epsilon^{2}$ (by putting one of the terms of (4) to the other side and taking the square). It can be shown that one of the roots is larger than $\left|\Delta_{l}\right|^{2}$ and thus must be rejected. Thus for a given phase difference, there is at most one pair $\left( \pm \epsilon_{b}\right)$ of bound states. The remaining root must be substituted back into eq (5) to check that it is indeed a solution. We found that as the ratio in gap magnitude increases, there is an increasing region near $\phi=0$ where the bound states cease to exist. 
Examples are shown in Fig. 1 and 2. The value of $\phi$ where the bound states first appear is given by $\phi_{c}=\cos ^{-1}\left(\left|\Delta_{l}\right| /\left|\Delta_{r}\right|\right)\left(\epsilon=\left|\Delta_{l}\right|\right.$ is a solution to eq (5) at $\left.\cos \phi=\left|\Delta_{l}\right| /\left|\Delta_{r}\right|\right)$. This critical phase difference $\phi_{c}$ is independent of $D$, and $\phi_{c} \rightarrow \pi / 2$ when $\left|\Delta_{l}\right|<<\left|\Delta_{r}\right|$.

To understand this $\phi_{c}$, it is convenient [11] to imagine two small normal regions between the two superconductors surrounding the barrier (thus a $\mathrm{S}_{l} \mathrm{~N}_{l} \mathrm{IN}_{r} \mathrm{~S}_{r}$ junction. Here $\mathrm{S}_{l, r}$ are the two superconductors, I the barrier and $\mathrm{N}_{l, r}$ are the two normal regions.). Quasiparticles and holes in $\mathrm{N}_{l, r}$ are transmitted and reflected by the barrier I while Andreev reflected if they incident on the superconductors. Recall that for a particle (hole) incident on a superconductor, the Andreev reflected hole (particle) acquires a phase $-\cos ^{-1}\left(\frac{\epsilon}{|\Delta|}\right)$ in addition to that from the phase of the order parameter $\mp \arg (\Delta)$. Now one can easily understand $\phi_{c}$ for the case with perfect transmission. In this case one can ignore I and identify $\mathrm{N}_{l, r}$. Let us take, without loss in generality, $\phi_{r}=\phi$ and $\phi_{l}=0$. One can readily see that one has an Andreev bound state at $\epsilon=\left|\Delta_{l}\right|$ at $\phi=\phi_{c}$ consisting of a hole propagating to the right and a particle propagating to the left. In this case there is no phase shift at the Andreev reflection at the left superconductor (since $\epsilon=\left|\Delta_{l}\right|$ ), while the two contributions at the reflection at the right superconductor cancels $\left(\phi_{r}-\cos ^{-1}\left(\frac{\epsilon}{\left|\Delta_{r}\right|}\right)=0\right)$.

Note that for the above case, the phase shift does not cancel for an incident particle (reflected hole) to the right superconductor. Since reflection at the barrier I can in general generate this amplitude, it may be thus surprising at first sight that the bound state at $\epsilon=\left|\Delta_{l}\right|$ still exists when $D \neq 1$. However, a careful analysis (along the lines of [11]) shows that the bound state still exists but with the amplitude of the right moving particle in $\mathrm{N}_{r}$ vanishes identically. The amplitude of the particle incident from the left of the barrier (in $\mathrm{N}_{l}$ ) and the amplitude of the particle incident from the right of it (in $\mathrm{N}_{r}$ ) are in such a ratio that the outgoing, right moving particle amplitude in $\mathrm{N}_{r}$ still vanishes exactly.

The supercurrent is given by

$$
I=-\frac{e}{2 \pi^{2} i \hbar} \int d \epsilon \frac{1}{4} \operatorname{Tr}_{4}\left\{\hat{\tau}_{3} \hat{d}^{R-A}\right\} f(\epsilon)
$$

where we have used the short-hand $\hat{d}^{R-A} \equiv \hat{d}^{R}-\hat{d}^{A}$ and $f(\epsilon)$ is the Fermi function. Explicitly, 
we find

$$
\begin{aligned}
I= & \frac{e D\left|\Delta_{r}\right|\left|\Delta_{l}\right| \sin \phi}{\hbar \epsilon_{b}}\left[\frac{1}{\left(1-\frac{D}{2}\right)\left(\frac{\alpha_{r}}{\alpha_{l}}+\frac{\alpha_{l}}{\alpha_{r}}\right)+D}\right] \tanh \left(\frac{\epsilon_{b}}{2 T}\right) \\
& +\frac{e D\left|\Delta_{r}\right|\left|\Delta_{l}\right| \sin \phi}{\pi \hbar} \int_{\left|\Delta_{l}\right|}^{\left|\Delta_{r}\right|} d \epsilon \frac{\left(1-\frac{D}{2}\right) \sqrt{\left|\Delta_{r}\right|^{2}-\epsilon^{2}} \sqrt{\epsilon^{2}-\left|\Delta_{l}\right|^{2}} \tanh (\epsilon / 2 T)}{\left(1-\frac{D}{2}\right)^{2}\left(\left|\Delta_{r}\right|^{2}-\epsilon^{2}\right)\left(\epsilon^{2}-\left|\Delta_{l}\right|^{2}\right)+\left(\frac{D}{2}\right)^{2}\left[\left|\Delta_{r}\right|\left|\Delta_{l}\right| \cos \phi-\epsilon^{2}\right]^{2}}
\end{aligned}
$$

where we have defined $\alpha_{l, r}=\sqrt{\left|\Delta_{l, r}\right|^{2}-\epsilon_{b}^{2}}$. The first term, $I_{b}$, due to the bound states, is to be included only when these states exist. $I_{b}$ can also be found from $-\frac{2 e}{\hbar} \frac{\partial \epsilon_{b}}{\partial \phi}$. Examples of $I_{b}$ were plotted in Fig 1 and 2. $I_{b}$, however, is not the only contribution to the current (as in the case for equal gaps). $\hat{d}^{R-A}$ is also finite for $\left|\Delta_{l}\right|<\epsilon<\left|\Delta_{r}\right|$ (though still vanishes for $|\epsilon|>\left|\Delta_{r}\right|$ ). This (and thus the second term of eq (7)) represents the contribution from continuum states with energies in the above range incident from the left superconductor and Andreev reflected from the right one. These states carry a finite current when $\phi \neq 0$. For low transmission, the continuum states contribution can actually dominate. Two examples of the various contributions to the current are shown in Fig. 3. It turns out that the dependence of the total current on $\phi$ is not much different from the case with equal gaps, except the magnitude is modified.

We finally turn to the current noise of our junction. We shall study the quantity [14]

$$
S_{a}(\omega) \equiv \int d t e^{i \omega t}\left(<\hat{I}(t) \hat{I}(0)>-<\hat{I}>^{2}\right)
$$

$S_{a}$ represents the spectral density for absorption by the junction of energy quanta $\omega . S_{a}$ can be written (c.f. [16]) in terms of the envelope functions [17] for the Green's function near the interface (see Ref [18]) as

$$
S_{a}(\omega)=\frac{e^{2}}{\hbar} \int \frac{d \epsilon}{2 \pi}(1-f(\epsilon+\omega)) f(\epsilon) K(\epsilon)
$$

where

$$
\begin{array}{r}
K(\epsilon) \equiv \frac{1}{2}\left\{\operatorname { T r } _ { 4 } \left[\hat{\tau}_{3} \hat{C}_{++}^{R-A}(\epsilon+\omega) \hat{\tau}_{3} \hat{C}_{++}^{R-A}(\epsilon)+\hat{\tau}_{3} \hat{C}_{--}^{R-A}(\epsilon+\omega) \hat{\tau}_{3} \hat{C}_{--}^{R-A}(\epsilon)\right.\right. \\
\left.\left.-\hat{\tau}_{3} \hat{C}_{+-}^{R-A}(\epsilon+\omega) \hat{\tau}_{3} \hat{C}_{-+}^{R-A}(\epsilon)-\hat{\tau}_{3} \hat{C}_{-+}^{R-A}(\epsilon+\omega) \hat{\tau}_{3} \hat{C}_{+-}^{R-A}(\epsilon)\right]\right\}
\end{array}
$$


This formula is valid on either side of the junction, and we shall choose to do this on the left (and correspondingly leave out the subscripts $l$ in $\hat{C}^{\prime}$ s.) $\hat{C}_{++}$and $\hat{C}_{--}$can be expressed in terms of $\hat{s}$ and $\hat{d}$ given earlier via $\hat{C}_{ \pm \pm}= \pm \frac{i}{2}+\frac{1}{4 \pi}\left(\hat{s}_{l} \pm \hat{d}\right) . \hat{C}_{+-}$and $\hat{C}_{-+}$are given by (c.f. [16], see also [18]) $\hat{C}_{+-}=\frac{i}{2} r^{*}\left(\hat{1}-\frac{i}{\pi} \hat{g}_{l, \infty}\right) / \mathcal{D}$ and $\hat{C}_{-+}=-\frac{i}{2} r\left(\hat{1}+\frac{i}{\pi} \hat{g}_{l, \infty}\right) / \mathcal{D}$. Here $r$ is the reflection coefficient for normal quasiparticles incident from the left of the barrier, and $|r|^{2}=1-D$.

With these $\hat{C}$ 's, the expression for $S_{a}$ can be written as eq (9) with $K=K_{1}+K_{2}$ where

$$
\begin{aligned}
K_{1}(\epsilon, \omega) \equiv & -4(1-D) \operatorname{Im}\left[\frac{1}{\mathcal{D}}\right]_{\epsilon+\omega} \operatorname{Im}\left[\frac{1}{\mathcal{D}}\right]_{\epsilon} \\
& +D^{2}\left\{\left(\tilde{\mu}_{l, \epsilon+\omega} \tilde{\mu}_{l, \epsilon}+\mu_{l, \epsilon+\omega} \mu_{l, \epsilon}\left|\Delta_{l}\right|^{2}\right)+(l \rightarrow r)\right\} \\
& +D(2-D)\left\{\left(\tilde{\mu}_{l, \epsilon+\omega} \tilde{\mu}_{r, \epsilon}+\tilde{\mu}_{r, \epsilon+\omega} \tilde{\mu}_{l, \epsilon}\right)+\left(\mu_{l, \epsilon+\omega} \mu_{r, \epsilon}+\mu_{r, \epsilon+\omega} \mu_{l, \epsilon}\right)\left(\left|\Delta_{l}\right|\left|\Delta_{r}\right| \cos \phi\right)\right\}
\end{aligned}
$$

and

$$
\begin{aligned}
K_{2}(\epsilon, \omega) \equiv & D^{2}\left\{\operatorname{Im}\left[\frac{\epsilon}{Q}\right]_{\epsilon+\omega} \operatorname{Im}\left[\frac{\epsilon}{Q}\right]_{\epsilon}\left(\left|\Delta_{r}\right|^{2}+\left|\Delta_{l}\right|^{2}-2\left|\Delta_{r}\right|\left|\Delta_{l}\right| \cos \phi\right)\right. \\
& \left.+\operatorname{Im}\left[\frac{1}{Q}\right]_{\epsilon+\omega} \operatorname{Im}\left[\frac{1}{Q}\right]_{\epsilon}\left(\left|\Delta_{r}\right|^{2}\left|\Delta_{l}\right|^{2} \sin ^{2} \phi\right)\right\}
\end{aligned}
$$

Here we have introduced the short hands $\mu_{l, r}(\epsilon)=\operatorname{Im}\left[\frac{1}{\sqrt{\left|\Delta_{l, r}\right|^{2}-\epsilon^{2}} \mathcal{D}(\epsilon)}\right]$ and $\tilde{\mu}_{l, r}(\epsilon) \equiv$

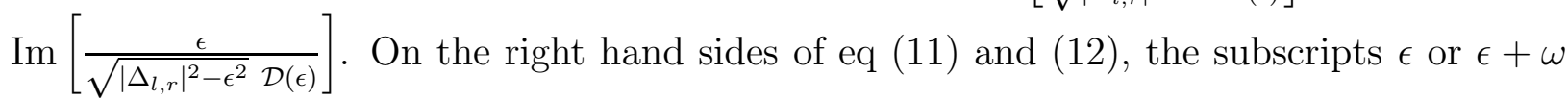
specify where the functions are to be evaluated and all $\epsilon$ 's are understood to be $\epsilon+i \eta$. Here $\eta$ is a damping coefficient. $[7,8]$

An example of the current-noise is as shown in Fig 4. At increasing gap ratios, $\epsilon_{b}$ moves towards the gap edge $\left|\Delta_{l}\right|$. Correspondingly the peaks at $\omega=0$ and $\omega=2 \epsilon_{b}$ decreases. When the bound states no longer exist, these two peaks are entirely absent and the noise becomes low for all frequencies below twice the smaller gap. This happens in particular for small phase differences and large gap ratios. Note also that the contribution from continuum states is strongly suppressed when $T<<\left|\Delta_{l}\right|$.

In conclusion, we have studied the Andreev bound states and supercurrent for a quantum point contact between two superconductors with different gap magnitudes. We showed in 
particular that the current noise can be qualitatively different from the case where the superconductors are identical.

This research was supported by the National Science Council of Taiwan grant number NSC90-2112-M-001-061 and NSC91-2112-M-001-063. 


\section{REFERENCES}

[1] See, e.g., Mesoscopic Electron Transport, ed. by L. L. Sohn, L. P. Kouwenhoven and G. Schön, NATO ASI series E, Vol. 345, Kluwer Academic Publishing, Dordrecht (1997)

[2] D. Averin and A. Bardas, Phys. Rev. Lett., 75, 1831 (1995)

[3] D. Averin and A. Bardas, Phys. Rev. B 53, R1705 (1996); D. V. Averin, A. Bardas and H. T. Imam, Phys. Rev. B 58, 11165 (1996)

[4] J. C. Cuevas, A. Martin-Rodero and A. Levy Yeyati, Phys. Rev. Lett., 82, 4086 (1999)

[5] M. C. Koops et al, Phys. Rev. Lett., 77, 2542 (2000)

[6] M. F. Goffman et al, Phys. Rev. Lett., 85, 170 (2000)

[7] D. Averin and H. T. Imam, Phys. Rev. Lett., 76, 3814 (1996)

[8] A. Martin-Rodero, A. Levy Yeyati and F. J. Garcia-Vidal, Phys. Rev. B, 53, R8891 (1996)

[9] Y. Naveh and D. V. Averin, Phys. Rev. Lett., 82, 4090 (1999)

[10] A. Furusaki and M. Tsukada, Physica B 165 \& 166, 967 (1990)

[11] C. W. J. Beenakker, Phys. Rev. Lett., 67, 3836 (1991)

[12] A. V. Zaitsev, Zh. Eksp. Teor. Fiz, 86, 1742 (1984) [ Sov. Phys. JETP 59, 1015 (1984)]

[13] S. K. Yip, J. Low Temp. Phys. 109, 547 (1997)

[14] We are following the suggestions of [15] to study an asymmetrically defined currentcurrent correlation function which directly relates to a certain (here absorption) processes. The spectral density $S(\omega)$ defined in, e.g., [7] with $\hat{I}(t) \hat{I}(0)$ replaced by $\frac{1}{2}(\hat{I}(t) \hat{I}(0)+\hat{I}(0) \hat{I}(t))$ is given by $S(\omega)=\frac{1}{2}\left(1+e^{-\omega / T}\right) S_{a}(\omega)$.

[15] U. Gavish, Y. Levinson and Y. Imry, in "Interacting Electrons in Nanostructures", ed. by R. Haug and H. Schoeller, Springer, Berlin (2001) 
[16] V. A. Khlus, Zh. Eksp. Teor. Fiz, 93, 2719 (1987) [ Sov. Phys. JETP 66, 1243 (1987)]

[17] A. J. Millis, D. Rainer and J. A. Sauls, Phys. Rev B, 38, 4504 (1988)

[18] Here, for completeness, we provide the definitions for the envelope functions $\hat{C}$ 's and explain how they can be obtained. Near the interface the Nambu Green's function $\hat{G}$ can be expressed in the form [17]

$$
\hat{\tau}_{3} \hat{G}\left(x, t, x^{\prime}, t^{\prime}\right)=\frac{1}{p / m} \sum_{j, j^{\prime}} \hat{C}_{j j^{\prime}}\left(x, t, x^{\prime}, t^{\prime}\right) e^{i p\left(j x-j^{\prime} x^{\prime}\right)}
$$

such that $\hat{C}_{j j^{\prime}}$ are slowly varying functions of $x$ and $x^{\prime}$. Here $j, j^{\prime}$ are \pm . The $\hat{C}_{j j^{\prime}}(\epsilon)$ are the Fourier transform ( in time) of $\hat{C}_{j j^{\prime}}(t)$ which are in turn given by $\lim _{x^{\prime} \rightarrow x+0_{+}} \hat{C}_{j j^{\prime}}\left(x, t, x^{\prime}, 0\right)$.

$\hat{C}_{++}$and $\hat{C}_{--}$were already given in text. From eq (54) of [17] ( with $l$ and $r$ interchanged), we have

$$
r \hat{C}_{l,+-}+r^{*} \hat{C}_{l,-+}=\hat{C}_{l,++}+|r|^{2} \hat{C}_{l,--}-D \hat{C}_{r,++}
$$

We can eliminate $\hat{C}_{l,+-}$ by multiplying this equation by $\left(\hat{C}_{l,++}-i\right)$, since $[17]\left(\hat{C}_{l,++}-\right.$ i) $\hat{C}_{l,+-}=0$. We thus obtain an expression for $\left(\hat{C}_{l,++}-i\right) \hat{C}_{l,-+}$. On the other hand $\left(\hat{C}_{l,--}+i\right) \hat{C}_{l,-+}=0$. Subtracting these two equations, we obtain an equation from which we can solve for $\hat{C}_{l,-+}$, since $\left(\hat{C}_{l,++}-\hat{C}_{l,--}-2 i\right)$ is invertible (c.f. [13]). $\hat{C}_{l,+-}$ can be obtained in a similar manner. 


\section{FIGURES}

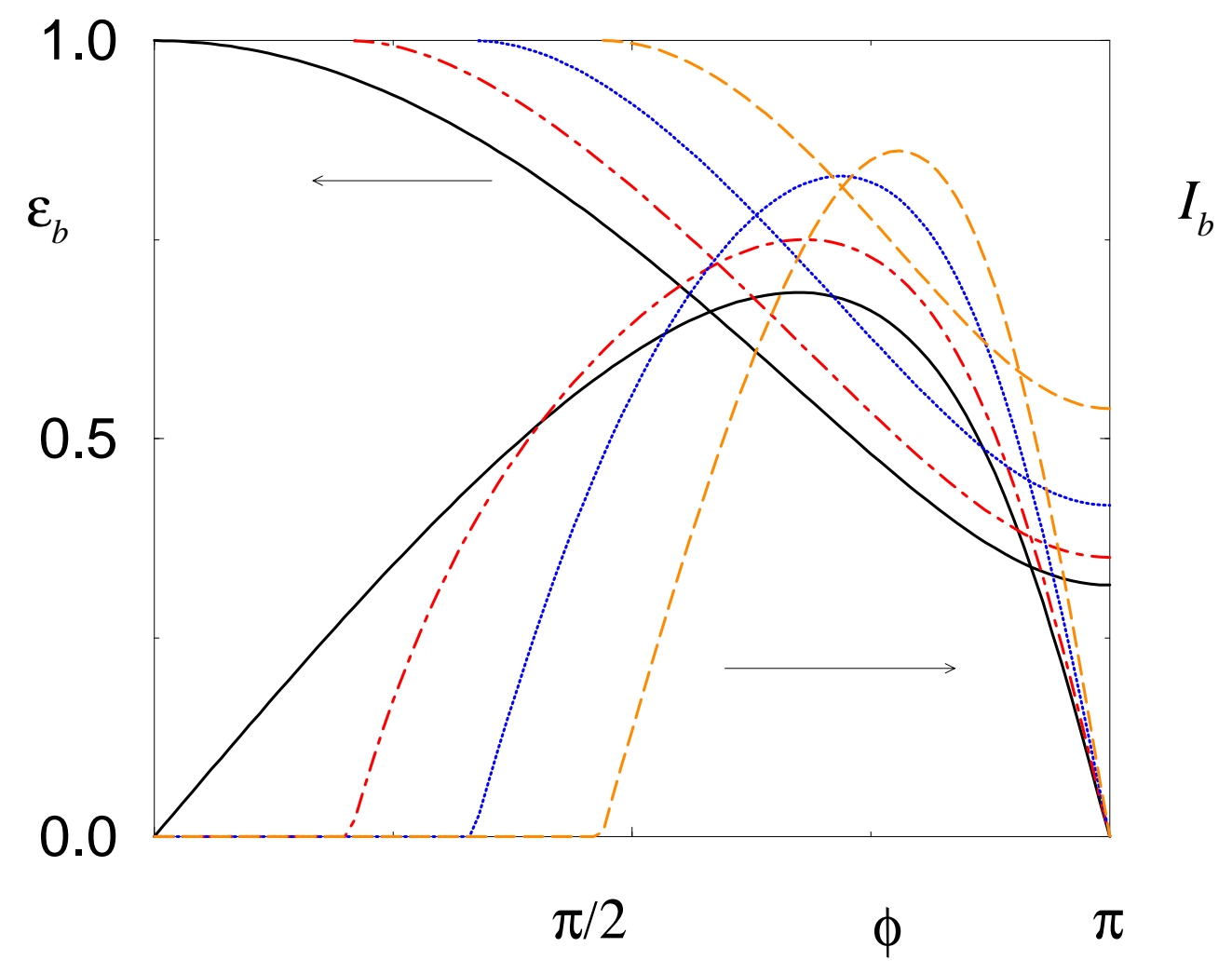

FIG. 1. Bound state energy $\epsilon_{b}$ (in unit of $\left|\Delta_{l}\right|$, left axis), and the bound state contribution to the current (in unit of $e\left|\Delta_{l}\right| / \hbar$, right axis) as a function of $\phi . D=0.9$, while $\left|\Delta_{r}\right| /\left|\Delta_{l}\right|$ 's are given by 1.0 (full line), 1.25 (dot-dashed), 2.0 (dotted) and 10 (long-dashed). 


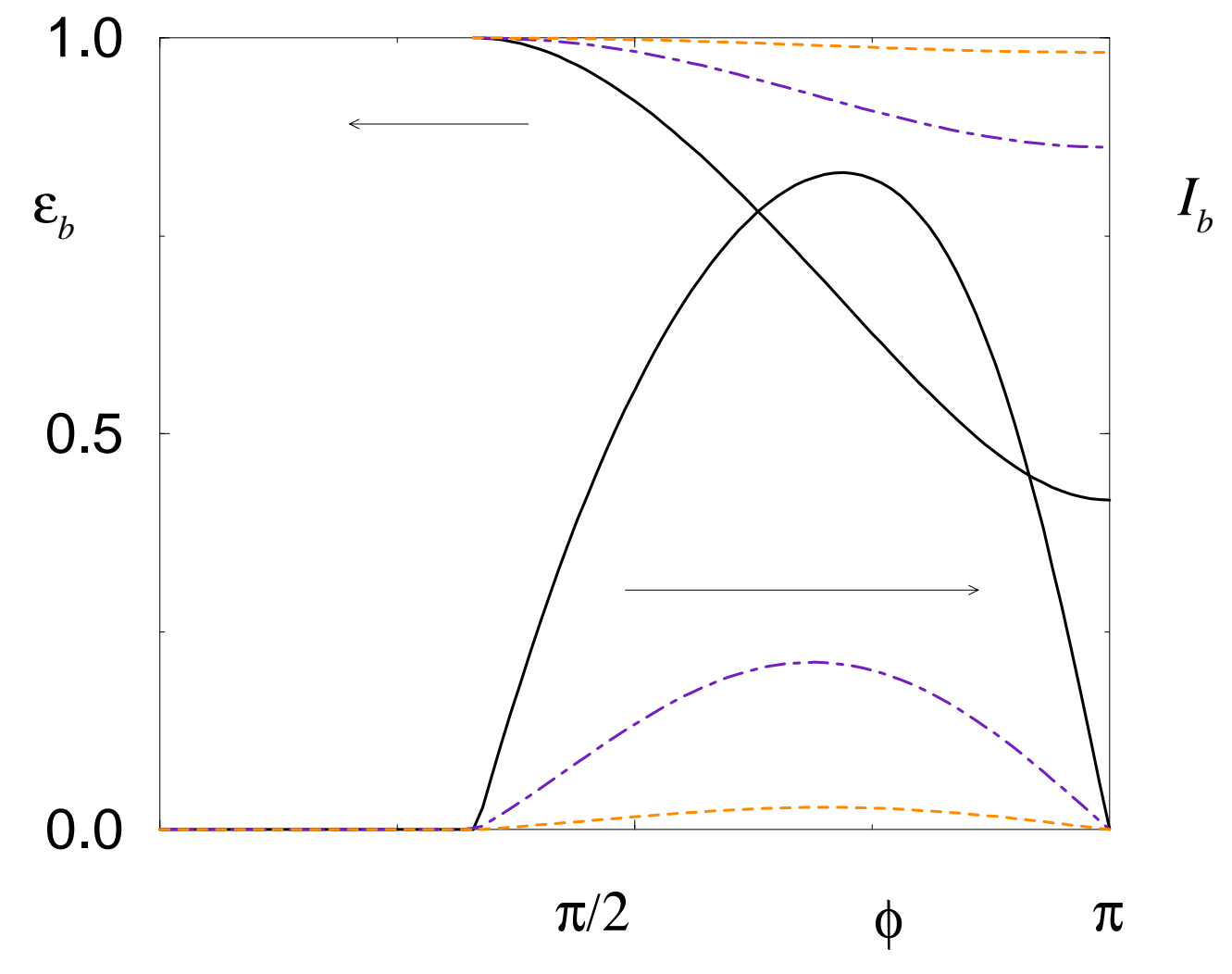

FIG. 2. Bound state energy $\epsilon_{b}$ (in unit of $\left|\Delta_{l}\right|$, left axis), and the bound state contribution to the current (in unit of $e\left|\Delta_{l}\right| / \hbar$, right axis) as a function of $\phi .\left|\Delta_{r}\right| /\left|\Delta_{l}\right|=2.0$, while $D$ 's are given by 0.9 (full line), 0.5 (dot-dashed), and 0.2 (long-dashed). 


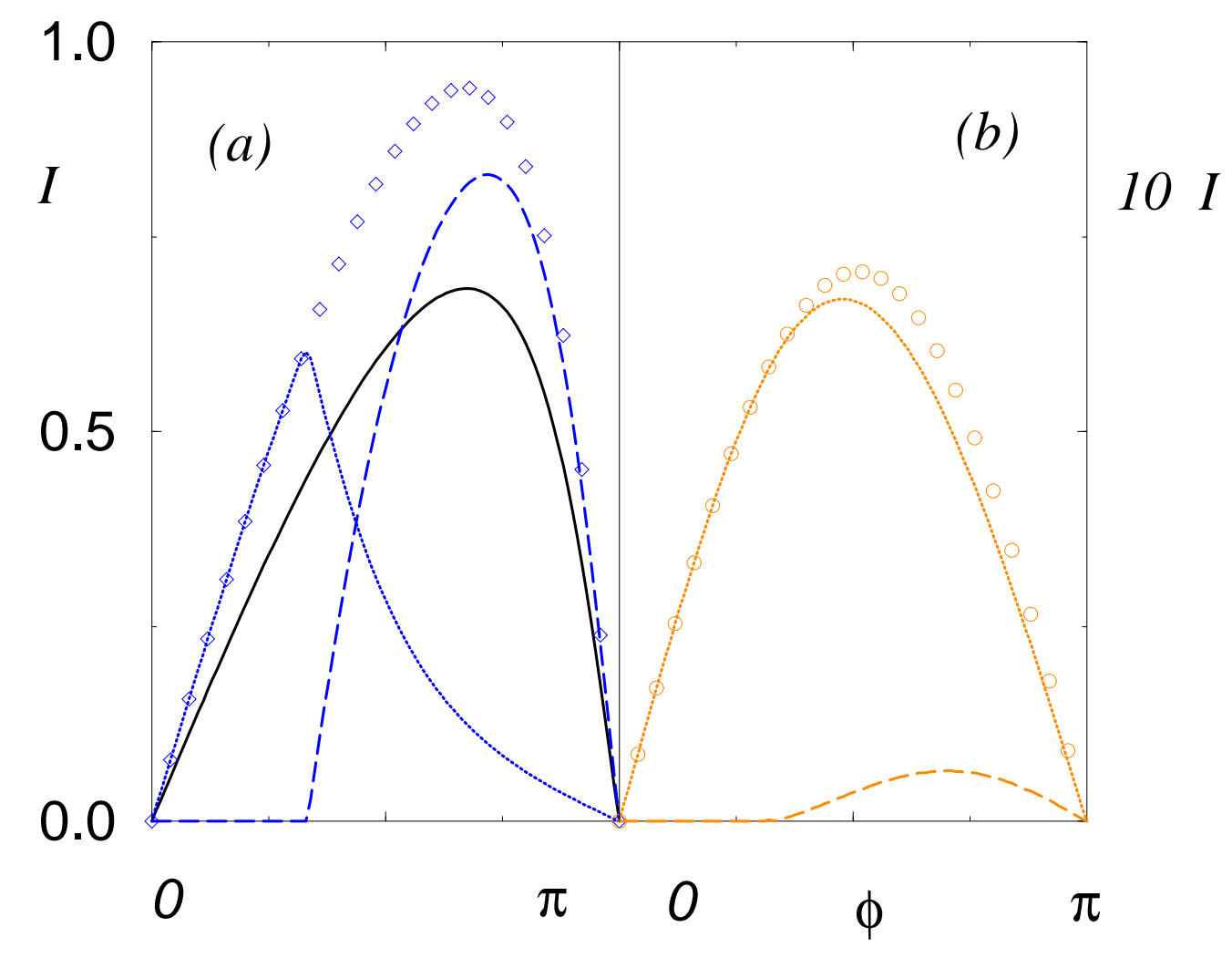

FIG. 3. Current-phase dependence for (a): $D=0.9,\left|\Delta_{r}\right| /\left|\Delta_{l}\right|=2.0 . T=0$, and currents are in units of $\frac{e\left|\Delta_{l}\right|}{\hbar}$. Long-dashed: $I_{b}$, dotted: continuum contribution, symbols: total. The current for $\left|\Delta_{r}\right|=\left|\Delta_{l}\right|$ is also shown (full line) for comparison. (b): Same as (a) except $D=0.1$. Note the difference in scale for $I$. 


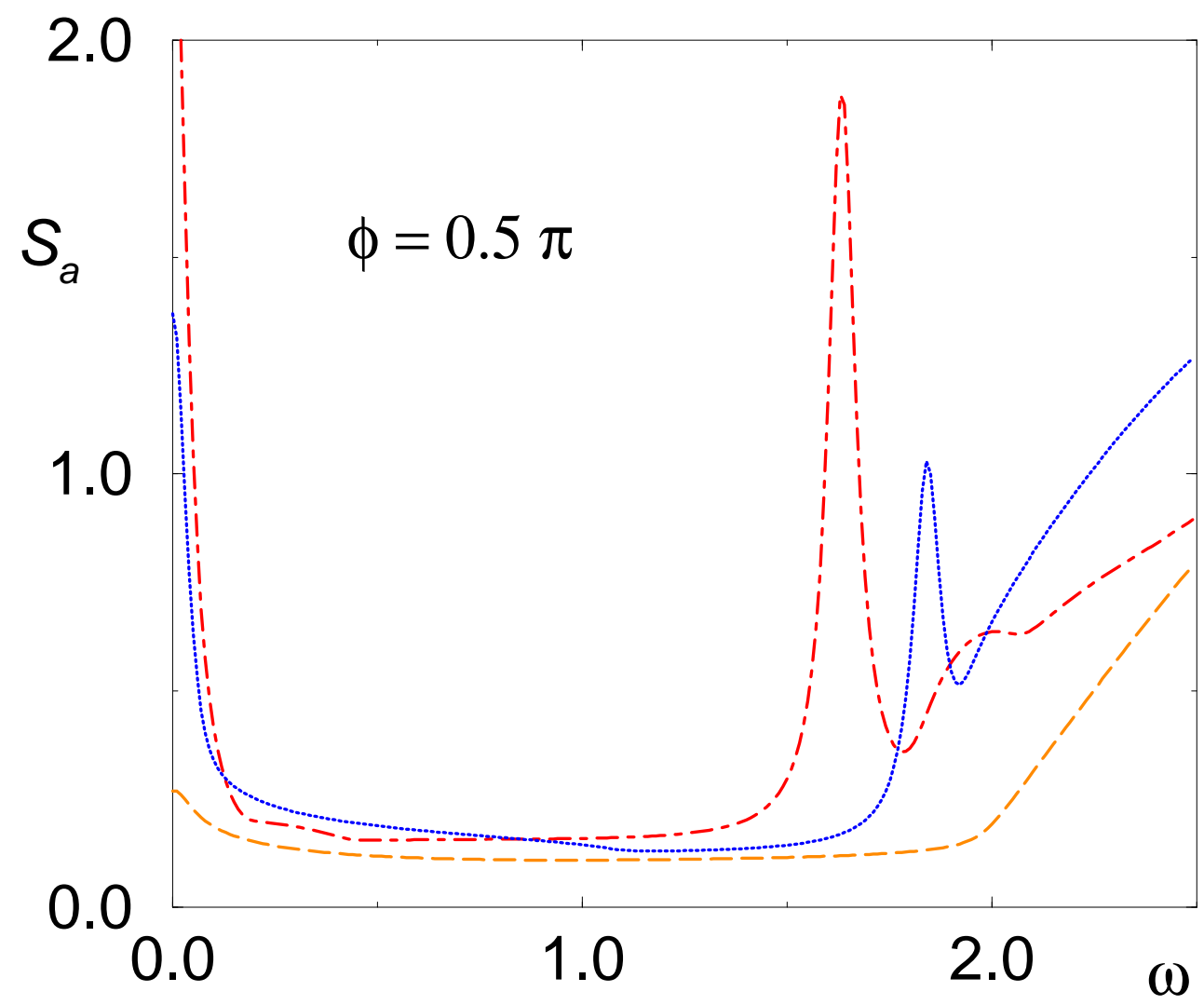

FIG. 4. $S_{a}(\omega)$ for $D=0.9, \phi=0.5 \pi, T /\left|\Delta_{l}\right|=0.3$, and damping coefficient $\eta=0.02\left|\Delta_{l}\right|$. $\left|\Delta_{r}\right| /\left|\Delta_{l}\right|$ are given by, dot-dashed: 1.0, dotted: 2.0, long dashed 10.0. $S_{a}$ in unit of $\frac{e^{2}\left|\Delta_{l}\right|}{\hbar}, \omega$ in unit of $\left|\Delta_{l}\right|$. 